JOURNAL CLUB

\title{
Nicotine replacement therapy in pregnancy
}

Cigarette smoking is a major modifiable cause of adverse pregnancy outcomes. Nicotine-replacement therapy (NRT) is recommended in guidelines, but its efficacy and safety during pregnancy is uncertain.

This multicentre double-blind trial recruited 1050 pregnant participants who were in their 12-24 weeks' gestation periods. All of them smoked $\geq 5$ cigarettes per day (median 20). Participants were randomly assigned NRT $(15 \mathrm{mg} / 16 \mathrm{~h})$ or placebo patches for 8 weeks. All individuals received behavioural support given the sound evidence base in pregnancy.

The primary outcome was abstinence from individually set quit dates through to delivery. Self-reported smoking status was validated using salivary cotinine and exhaled carbon monoxide concentration. NRT significantly increased quit rates at 1 month, but by term there was no difference between abstinence rates in the NRT (9.4\%) and placebo $(7.6 \%)$ arms $(\mathrm{OR}=1.26$ (95\% CI 0.82 to 1.96$))$.

Rates of adverse pregnancy and birth outcomes (such as stillbirth, miscarriage and low birth weight) were no different in the two groups.

A key finding was low adherence to therapy: $7.2 \%$ in the intervention group and $2.8 \%$ in the placebo group used patches for $>1$ month. Poor adherence due to perceived lack of efficacy has been seen repeatedly in other trials of NRT, and this study may have been particularly affected due to the increases in nicotine and cotinine clearance during pregnancy. Moreover, the low adherence rate limits the inference that can be made from the data on adverse outcomes.

This study, taken together with previous investigations, suggests NRT should not be routinely recommended in pregnancy.

- Coleman T, Cooper S, Thornton JG, et al; Smoking, Nicotine, and Pregnancy (SNAP) Trial Team. A randomized trial of nicotine-replacement therapy patches in pregnancy. N Engl J Med 2012;366:808-18.

Thomas D Stringfellow, John D Blakey

Correspondence to Dr John D Blakey, Clinical Lecturer, Respiratory Medicine, Clinical Sciences Building, City Hospital Campus, Nottingham NG5 1PB, UK; john.blakey@nottingham.ac.uk

Thorax 2012;68:110. doi:10.1136/thoraxjnl-2012-202056 